\title{
Validating of the pre-clinical mouse model for metastatic breast cancer to the mandible
}

\author{
Young Sun HWANG ${ }^{1 *}$, Sang-Sun HAN ${ }^{1 *}$, Ki-Rim KIM², Ye-Jin LEE ${ }^{1}$, Sun-Kyung LEE ${ }^{3,4}$, Kwang-Kyun PARK ${ }^{3,4}$, Won- \\ Yoon CHUNG ${ }^{3,4}$ \\ *These authors contributed equally to this work
}

\author{
1- Department of Dental Hygiene, College of Health Science, Eulji University, Seongnam, Republic of Korea. \\ 2- Department of Dental Hygiene, Kyungpook National University, Sangju, Republic of Korea. \\ 3- Oral Cancer Research Institute, Department of Oral Biology, and BK21 PLUS Project, Yonsei University College of Dentistry, Seoul, Republic of Korea. \\ 4- The Applied Life Sciences, Graduate School, Yonsei University, Seoul, Republic of Korea.
}

Corresponding address: Young Sun Hwang - Department of Oral Hygiene, College of Health Science, Eulji University, Seongnam, Republic of Korea - Phone: +82 0222283058 - Fax: +82 23647113 - e-mail: kiteys@eulji.ac.tr

Submitted: May 1, 2014 - Modification: September 15, 2014 - Accepted: October 7, 2014

\section{ABSTRACT}

\begin{abstract}
M etastatic breast carcinoma has a great tendency to spread to the mandible. It is concomitantly associated with bone destruction, food intake disorder, and a poorer prognosis. Appropriate animal models need to be developed for a better understanding of the mechanisms underlying the metastatic process of breast cancer cells to mandible and to test the effects of potential lead compounds. Here, we assessed the metastasis model of intracardiac injection using luciferase-transfected metastatic breast cancer cells (MDAMB-231 ${ }^{\text {Luc }}$ ) by determining the incidences of metastasis, $\mu \mathrm{CT}$ images, and histopathological results. A high bioluminescence signal mainly detected mandibular lesions with less frequent distal femora and proximal tibiae lesions. Extensive mandibular bone destruction occurred in nude mice grafted with metastatic breast cancer cells. This type of animal model might be a useful tool in assessing therapeutic implications and the efficacy of anti-cancer drugs for osteolytic cancers.
\end{abstract}

Keywords: Breast cancer. Mandible. Osteolysis. Xenograft. Luminescence.

\section{INTRODUCTION}

Bone is one of the most common sites for metastasis $^{29}$. Bone metastases represent a major complication of malignant tumors. Metastatic tumors to the mandibles not a uncommon phenomenon and account for approximately $1 \%$ of all malignant oral tumors ${ }^{27}$. Metastatic tumors in the mandible are more frequently reported than those in the oral mucosa. In particular, breast carcinoma metastasizes to the mandible three times as often as any other malignant tumor ${ }^{1}$. However, the pathogenesis of the metastatic process in the mandible remains unclear. Metastatic tumors to the oral region are challenging and difficult to diagnose because of their relative infrequency. Therefore, they should be considered in the differential diagnosis of inflammatory and reactive lesions that are common to the oral region.

In the meantime, most of the currently available metastatic animal models of human breast tumor xenografts using orthotopic transplantation have only a limited success rate in most trials. The intratibia inoculation of breast cancer as a cancer-bone metastasis model fails to address certain aspects of the clinical situation and does not precisely reflect the characteristics of metastatic breast cancer ${ }^{8,13}$. Appropriate animal models are currently insufficient for the research before clinical trials. Thus, a feasible and adequate metastatic animal model needs to serve as a suitable tool to help precisely understand the molecular mechanism of mandible bone metastasis of breast cancer and to develop new therapeutic agents against metastatic breast cancer to bone.

In this study, we assessed the metastasis model of intracardiac injection using luciferase-transfected metastatic breast cancer cells, demonstrated its value as a useful in vivo tool for metastatic breast cancer to mandible bone, and examined the 
histopathological aspect. This type of animal model approach might have the additional advantage of potentially accelerating the biological discovery process.

\section{MATERIAL AND METHODS}

\section{Material and reagents}

Dulbecco's modified Eagle's medium (DMEM), Hank's balanced salt solution (HBSS), fetal bovine serum (FBS), antibiotic-antimycotic mixture containing $100 \mathrm{U} / \mathrm{ml}$ penicillin and $100 \mu \mathrm{l} / \mathrm{ml}$ streptomycin, phosphate-buffered saline (PBS), and $0.25 \%$ trypsin-EDTA were purchased from Gibco BRL Co. (Rockville, MD, USA). D-Luciferin potassium salt was obtained from Goldbio Technology (St. Louis, MO, USA). DMSO and all other chemicals were purchased from Sigma-Aldrich (St. Louis, MO, USA). All reagents used in this research were of analytical grade.

\section{Cell culture}

The human mammary carcinoma cell line MDA-MB-231 was obtained from the Korean Cell Line Bank (Seoul, Korea) and cultured in DMEM supplemented with $10 \%$ FBS at $37^{\circ} \mathrm{C}$ under a humidified atmosphere of $5 \% \mathrm{CO}_{2}$.

\section{Luciferase vector construction}

To generate the lentiviral particles with lentiviral vector-based luciferase, firefly luciferase gene from Photinus pyralis was amplified by polymerase chain reaction (PCR)-based methods using PTALLuc vector (Clontech, Palo Alto, CA, USA) as a template, followed by subcloning into the pLenti6/ V5 Directional TOPO Cloning vector in ViraPower ${ }^{\mathrm{TM}}$ Lentiviral Expression Systems (Invitrogen, Carlsbad, CA, USA). pLenti6/V5-Luc plasmid was subjected to DNA sequencing analysis to confirm a successful construction. Lentivirus particles were produced by cotransfecting the 293FT producer cell line with pLenti6/V5-Luc plasmid and the ViraPowerTM Packaging Mix. The cells were transducted using $2 \times 10^{7}$ lentiviral particles with transduction enhancer Polybrene at $10 \mathrm{mg} / \mathrm{ml}$ to establish luciferasetransfected MDA-MB-231 (MDA-MB-231 $1^{\text {Luc+ }}$ ) stable cells. Blasticidine $(10 \mu \mathrm{g} / \mathrm{ml})$ was added to select stably transduced cells. Blasticidine-resistance clones exhibited the V5 epitope detection against anti-V5 antibody by western blot analysis and revealed the maximum level of luciferase activity using a microplate spectrofluorometer (Molecular Devices, Palo Alto, CA, USA).

\section{Animals}

Female Balb/c nu/nu mice ( 5 weeks of age) were purchased from Nara Bio Inc. (Seoul, Korea) and maintained at $20-22^{\circ} \mathrm{C}$ on a $12 \mathrm{~h}$ light/dark cycle.
All animal studies were performed in accordance with experimental protocols that were approved by the animal ethics committee of Yonsei University College of Dentistry.

\section{Animal model of the metastatic breast} cancer to the mandible

Mice were anesthetized by intramuscular injection of a mixture with $30 \mathrm{mg} / \mathrm{kg}$ Zoletil (Verbac, Carros, France) and $10 \mathrm{mg} / \mathrm{kg}$ xylazine (Bayer Korea, Seoul, Korea). MDA-MB-231 Luc+ cells $\left(1 \times 10^{6}\right.$ cells $/ 0.1 \mathrm{ml}$ in HBSS) were injected into the left cardiac ventricle of nude mice with a 26-1/2 gauge needle according to the modification of the method $^{3}$. Metastatic progression in nude mice was shown with bioluminescence imaging on 6 weeks after intracardiac injection. Then all animals were euthanized by $\mathrm{CO}_{2}$ asphyxiation and histological analysis was performed.

\section{Bioluminescent imaging}

Bioluminescent in vivo imaging was detected with a highly sensitive CCD camera mounted in a light-tight specimen box (IVIS-200'TM, Xenogen, Caliper Life Sciences, Alameda, CA, USA). Animals were given the substrate $D$-Luciferin potassium salt by intraperitoneal injection of $150 \mathrm{mg} / \mathrm{kg}$ in Dulbecco's Phosphate Buffered Saline and then they were anesthetized with $1-3 \%$ isoflurane gas. Ten min after the D-Luciferin injection, mice were placed onto the warmed stage inside the light-tight camera box with continuous exposure to $1-2 \%$ isoflurane gas.

\section{$\mu$ CT analysis}

The mandibles of the nude mice were scanned using a Skyscan 1076 high-resolution $\mu \mathrm{CT}$ with 100 $\mathrm{Kv}, 140 \mathrm{~mA}$ current, rotation step $0.6^{\circ}$ and camera pixel size 35 mm (SkyScan, Antwerpen, Belgium). NRecon and CT analyzer (CTAn) software (SkyScan) were used to analyze the structure of the sample. Two-dimensional images were used to generate three-dimensional (3-D) reconstructions with the software. The bone volume (BV) was calculated from the processed images shown in 3-D image using CTAn software with the same diameter given.

\section{Hematoxylin and eosin (H\&E) staining}

The tissues were fixed in $10 \%$ buffered formalin solution and embedded in paraffin. Tissue sections ( $4 \mu \mathrm{m}$ thickness) were sectioned using a Leica 2135 microtome, deparaffinized in xylene, rehydrated through an alcohol series, and stained with H\&E for histopathology. Then, tissues were dehydrated and mounted. 


\section{Statistical analysis}

The statistical analysis was conducted using InStat $^{\mathrm{TM}}$ statistical software (GraphPad Software, Inc., San Diego, CA, USA), and results are expressed as mean \pm standard error. The statistical significance of differences between groups was analyzed via repeated measures of Student's paired and unpaired $t$-tests. Asterisks were used to graphically indicate statistical significance. $P$ values of $<0.05$ were considered significant.

\section{RESULTS}

Mouse model for metastatic breast cancer to the mandible

MDA-MB-231 Luc+ cells were inoculated into the left ventricle of mice and the metastatic progression of breast cancer was tracked through bioluminescence imaging using an IVIS Imaging System (Figure 1). Metastasis to mandible were detected in all mice injected with MDA-MB-231 $1^{\text {Luc }}$ cells $(n=250)$. A high level of total photon flux was observed following the injection of MDA-MB-231 Luc+ cells $(P<0.001)$. Multiple organ metastases were frequently observed in distal femora and proximal tibiae. The high bioluminescence signal was also mainly observed in mandibles.

\section{Mandible bone analysis using $\mu$ CT}

The $\mu \mathrm{CT}$ images afforded a nondestructive visualization of the changes that occurred over the entire mandible. Osteolytic lesions of mice were analyzed by radiography at 6 weeks after cancer cell injection. On the mediolateral radiograph, an extensive bone destruction was observed around the mandibular bone in mice injected with MDA-

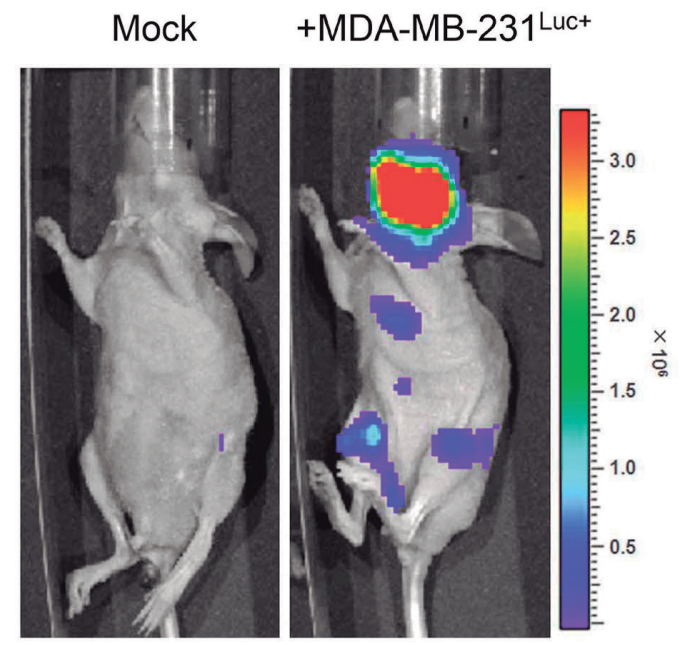

MB-231Luc+ cells (Figure 2A). Osteolytic lesions occurred in both cancellous and cortical bones on vertical projection (slice) (Figure $2 A$ ) and 3-D reconstruction images (Figure $2 \mathrm{~B}$ ). The bone volume was determined from the processed images shown in Figure 2B using CTAn software with the same diameter given as where bioluminescence signal and osteolytic site were overlapping (Figure 2C). The total bone volume was decreased about $28.9 \%$ in mice injected with MDA-MB-231Luc+ cells. The H\&E staining that tumour cells infiltrated into the mandibular region and thereby induced bone destruction (Figure 2D).

\section{DISCUSSION}

Carcinomas are malignant neoplasms derived from epithelial cells which are surrounded by stroma that interacts with cancer cells to modulate cell growth, migration, invasion, and tissue-specific gene expression. Recently studies have shown that interactions of tumor cells with their adjacent microenvironment are intimately associated with tumor growth and the development of metastasis. Therefore, appropriate in vivo animal models are required to study interactions between tumor cells and their environment. In addition, a feasible animal model is critical to test the effects of potential lead compounds.

Cancer is a complex disease in which cells develop aberrant proliferation and survival. The progression of metastasis severely increasis morbidity and eventually mortality. Metastatic tumors to the oral region are infrequent and account for approximately $1 \%$ of all malignant oral tumors ${ }^{27}$. However, metastatic tumors to the mandibular bone are not a rare phenomenon. Many

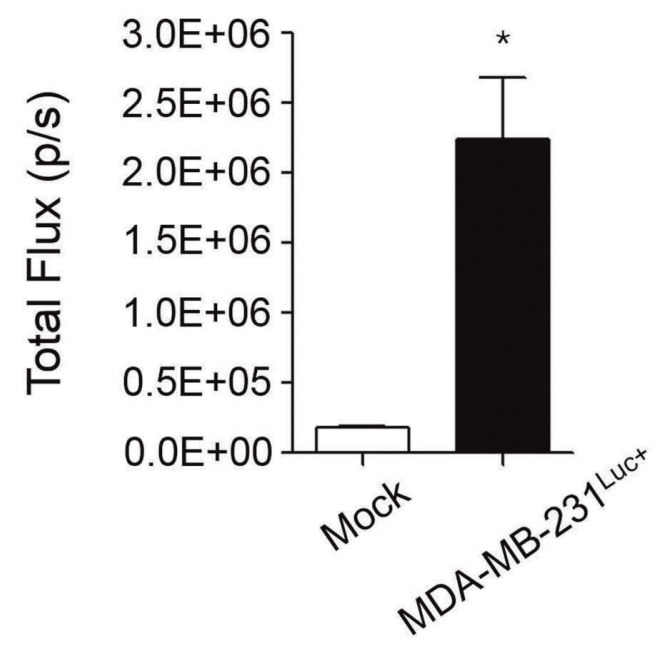

Figure 1- Mouse model for metastatic breast cancer to the mandible. The growth and part of the metastatic spread (left) was detected by bioluminescence imaging after the injection of cancer cells. The formed metastases were quantified by measuring total photon flux per second (right). Data are expressed as the mean \pm standard error (SEM). ${ }^{*} P<0.001$ versus Mock group 
cases with metatastic breast carcinoma to the mandible have been reported and their metastases are well documented. Metastases in the oral region can occur in oral soft tissues or the mandibular bone. Metastatic tumors in the mandibular bone are more frequently reported than those in the oral mucosa. Primary sites are usually the breast, lung, intestine, ovaries, prostate, thyroid, kidney, testes, uterine cervix, and adrenal glan $\mathrm{ds}^{9,11,12,14,15,17,19,23,24,27,30-32}$. Many authors reported that breast cancer metastasis to the oral cavity is more frequent for a disseminated tumor to involve the mandible rather than the maxilla1,21. Breast carcinoma is commonly osteolytic with an indistinct outline, but it is osteoblastic in $10 \%$ and mixed in type in another $10 \%$ of cases. Hormone therapy and sterilization may often result in the conversion of osteolytic lesions to the osteoblastic type ${ }^{2}$. Taken together, breast carcinoma has a great tendency to be spread by metastasis, thus it is a common clinical problem associated with bone destruction and a poorer prognosis. Breast carcinoma metastasizes to the mandibles three times as often as any other malignant tumor ${ }^{22}$. Also it has been reported that

A Mock

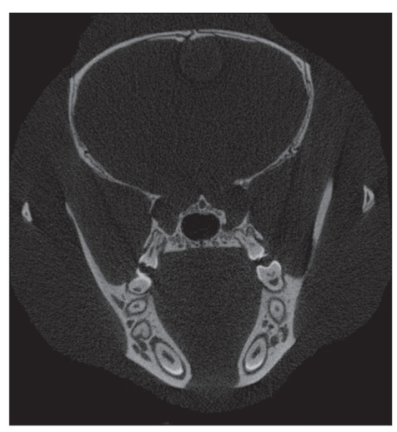

Mock

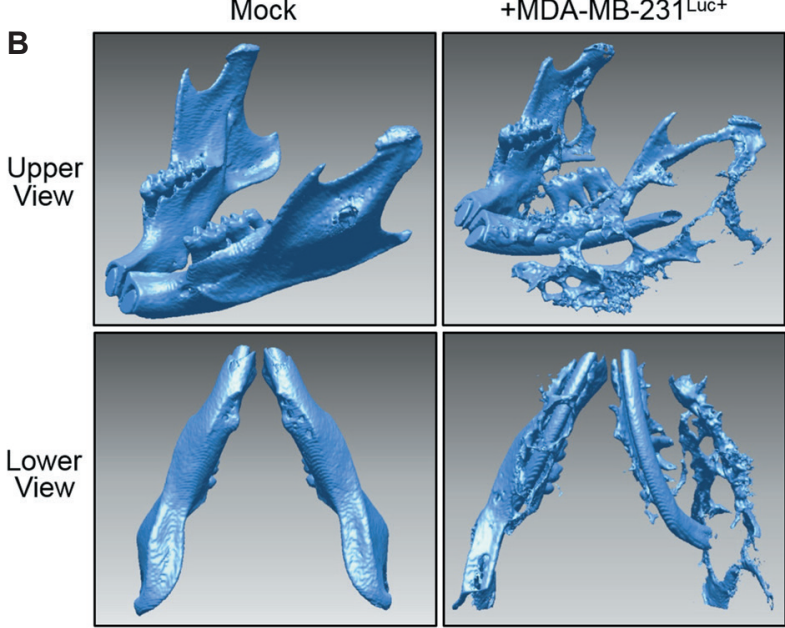

periodontal diseases are associated with breast cancer $^{28}$. Therefore, a metastasis of an aggressive breast cancer to the mandible has a tendency to increase. However, the complete understanding is lacking about the process how breast cancer metastasizes to the mandibles with the most frequency at the cellular level. Consequently, diversified insights into the molecular mechanism of breast cancer metastasis to the mandible are necessary to devise preventive and therapeutic approaches. An appropriate animal model might have an additional advantage to understand the mechanisms underlying the metastatic process and to test the effects of potential lead compounds.

Due to the technical complication, there is a low success rate in most trials due to the susceptibility of bacterial contamination and the weak cellular activity of primary tumors as opposed to metastatic tumors ${ }^{7,20}$. Human tumor xenografts using the orthotopic transplantation of histologically intact tissues of several carcinomas have not been adopted for a metastatic animal model although these models represent the entire environment that occurs during cancer progression ${ }^{16}$. Thus,

C
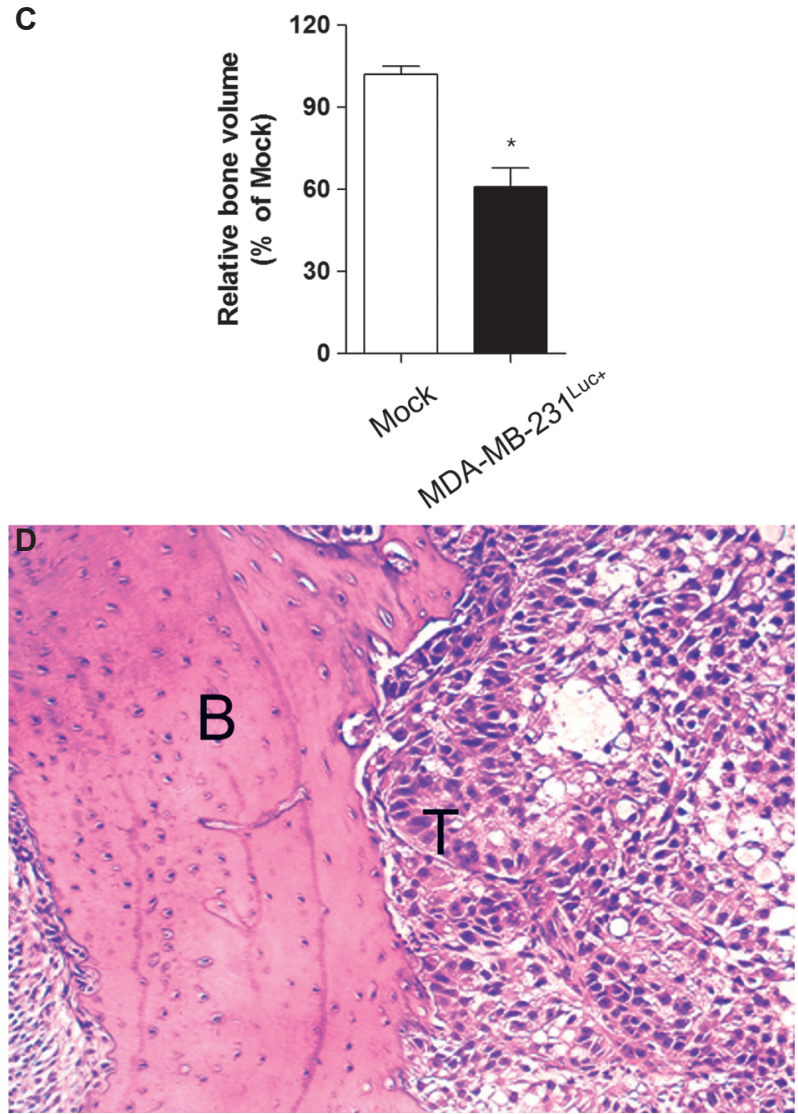

Figure 2- Mandible bone analysis. Radiographic images of mandibles in mice were scanned by $\mu \mathrm{CT}$ at 6 weeks. The osteolytic lesions were analyzed on vertical projection (slice) (A); and 3-D reconstruction images (B; upper and lower view each). The bone volume was determined from processed images shown in 3-D images (B); with the same given diameter (C). Data are expressed as the mean \pm standard error (SEM). ${ }^{*} P<0.01$ versus Mock group. Hematoxylin \& eosin staining around the mandibular region was performed and the tumor-induced osteolytic site was observed. (original magnification $\times 100)(B$; bone, $\mathrm{T}$; tumor) (D) 
xenograft models have been used to study the development and metastasis of human carcinomas. The xenograft animal model with a cultured cancer cell line is technically simple and reflects more precisely the morphology and character of their respective original tumor. Nevertheless, there is no single animal model that precisely replicates all of the events and circumstances that occur during cancer progression. The most widely used model for cancer development and behavior research is the flank model. However, not all transplanted cancer cells develop tumors at an atopic site due to the different cancer environments and no spontaneous metastasis does occur ${ }^{4}$. Also in case of a mammary fat pad xenograft, breast cancer development has shown a low success rate in most trials due to the technically limited occurrence of mammary glands in neonatal mice. Also, an intra-tibia inoculation of breast cancer is frequently applied as a cancer-bone metastasis model. In this model, cancer cells are inserted extra-capsulary through the tibial crest, epiphysis, and growth plate and then cells are sequentially injected into the bone marrow space. The intra-tibia model does not precisely reflect the characteristics of bone-invasive OSCC ${ }^{8,13}$. Thus, it fails to address certain aspects of the clinical situation. This model is not suitable to elucidate the cellular and molecular mechanisms of metastatic breast cancer to mandible and could not serve as suitable tool in the development of new therapeutic agents against metastatic breast cancer to bone.

Triple-negative breast cancer (TNBC), as defined by the absence of estrogen receptor, progesterone receptor, and human epidermal growth factor receptor 2 (HER2/neu) expression, can be particularly aggressive, and more likely to recur than other subtypes of breast cancer. MDA-MB-231 cell is triple-negative and preferentially metastasizes to the bone ${ }^{5}$. Particularly, metastatic MDA-MB-231 breast cancer cells have a clinical tendency to be spread to mandible. Breast carcinoma metastasizes to the mandibles three times as often as any other malignant tumor ${ }^{12}$. In many types of animal models including tibia, MDA-MB-231 cells led to osteolytic bone destruction, reproducibly. Therefore, MDAMB-231 cell is widely used for TNBC studies. However, a complete understanding of how breast cancer metastasizes to the mandibles with the most frequency at the cellular level is lacking.

Above all, in this study, we tried to find a proper animal model of metastatic breast cancer to the mandibular bone. Experimental metastases refer to the injection of tumor cells directly to the systemic circulation. Metastatic breast cancer cells have a great tendency to be spread to bone including mandible. Therefore, research groups have been adopting the intracardiac injection method for osteolytic metastasis model $17,18,25,26$.
Although this animal model accurately reflects the bone disease portion of the clinical disease, the complete mechanism of how breast cancer metastasizes to the mandibles with the most frequency at the cellular level is not clear. The active hematopoietic tissue is known as red bone marrow. Bones with red marrow are the favored sites for metastatic deposits ${ }^{17}$. Mainly in elderly persons, jawbones have little active marrow, while remnants of hematopoietic active marrow are detected in the posterior areas of the mandible and the hematopoietically active sites attract metastatic tumor cells. Meanwhile, the oral soft tissues have a rich network of capillaries which can entrap malignant cells. Proliferating capillaries display an uneven basement membrane, thereby increasing the penetration of tumor cells. In addition, inflammation plays an aggravative role in attracting metastatic cells to the gingiva9. In this study, firefly luciferase-expressing metastatic breast cancer cells (MDA-MB-231 $1^{\text {Luc }+}$ ) were injected into the left cardiac ventricle producing uniform mandibular bone metastases with complete success rate by ex vivo imaging and histological analysis. Osteolytic lesions were widely observed in both cancellous and cortical bones. The subsequent histological evaluation of tissue sections from the mandible confirmed the spontaneous tumor metastasis. In animal models, bioluminescent imaging is a useful and sensitive method for detection of primary cancer growth and for tracking the metastasis in animal models ${ }^{6,10}$. In addition, luciferase-expressing cells permit the real-time tracking of tumor sites in living animals.

\section{CONCLUSIONS}

This study verified the reproducible xenograft model of metastatic breast cancer to mandibular bone using intracardiac injection with MDA-MB$231^{\text {Luct. }}$ The development of breast cancer to mandible mouse model remains a challenge in the preclinical evaluation of therapies for human breast cancer. Now, the current xenograft model serves as suitable research tool and this application offers the preclinical assessment of anticancer therapies in vivo.

\section{CONFLICT OF INTEREST STATEMENT}

No conflict of interests.

\section{ACKNOWLEDGEMENTS}

This work was supported by a grant from the National R\&D Program for Cancer Control, Ministry of Health \& Welfare (1120190) and by the National Research Foundation of Korea (NRF) funded by the Ministry of Education, Science and Technology, Basic 
Science Research Program (2012R1A1A2040564), Republic of Korea.

\section{REFERENCES}

1- Adelson RT, DeFatta RJ, Miles BA, Hoblitt SL, Ducic Y. Metastatic breast cancer of the oral cavity. Am J Otolaryngol. 2005;26(4):279-81.

2- AshabYamin MR, Kalantarhormozi A, Hamdamjo F, Razi Z. Breast ductal carcinoma metastasis to jaw bones: a case report. Novel Biomed. 2014;2(1):31-5.

3- Bakewell SJ, Nestor P, Prasad S, Tomasson MH, Dowland $\mathrm{N}$, Mehrotra $\mathrm{M}$, et al. Platelet and osteoclast beta3 integrins are critical for bone metastasis. Proc Natl Acad Sci U S A. 2003;100(24):14205-10.

4- Broström LA, Crnalic S, Löfvenberg R, Boquist L, Stenling R. Growth patterns and cell kinetics of human osteosarcoma xenografts in serial passages in nude mice analyzed by in vivo labelling with iododeoxyuridine. J Cancer Res Clin Oncol. 1996;122:141-6.

5- Chavez KJ, Garimella SV, Lipkowitz S. Triple negative breast cancer cell lines: one tool in the search for better treatment of triple negative breast cancer. Breast Dis. 2010;32(1-2):35-48.

6- Craft N, Bruhn KW, Nguyen BD, Prins R, Liau LM, Collisson EA, et al. Bioluminescent imaging of melanoma in live mice. J Invest Dermatol. 2005;125(1):159-65.

7- Easty DM, Easty GC, Carter RL, Monaghan P, Butler LJ. Ten human carcinoma cell lines derived from squamous carcinomas of the head and neck. Br J Cancer. 1981;43(6):772-85.

8- Gregory LS, Choi W, Burke L, Clements JA. Breast cancer cells induce osteolytic bone lesions in vivo through a reduction in osteoblast activity in mice. PLoS One. 2013;8(9):e68103.

9- Hirshberg A, Leibovich P, Buchner A. Metastases to the oral mucosa: analysis of 157 cases. J Oral Pathol Med. 1993;22(9):38590.

10- Jenkins DE, Hornig YS, Oei Y, Dusich J, Purchio T. Bioluminescent human breast cancer cell lines that permit rapid and sensitive in vivo detection of mammary tumors and multiple metastases in immune deficient mice. Breast Cancer Res. 2005;7(4):R444-54. 11- Jham BC, Salama AR, McClure SA, Ord RA. Metastatic tumors to the oral cavity: a clinical study of 18 cases. Head Neck Pathol. $2011 ; 5(4): 355-8$.

12- Jia J, Chen XM, Sun ZJ, Zhang WF. Mandibular metastasis of nephroblastoma: a rare case. Int J Oral Maxillofac Surg. 2006;35(12):1160-1.

13- Jones MD, Liu JC, Barthel TK, Hussain S, Lovria E, Cheng D, et al. A proteasome inhibitor, bortezomib, inhibits breast cancer growth and reduces osteolysis by downregulating metastatic genes. Clin Cancer Res. 2010;16(20):4978-89.

14- Karimi M, Eshghi P. Unusual lymphoblastic leukemia/lymphoma in Eastern Iran. Indian J Pediatr. 2006;73(7):619-22.

15- Kechagias N, Ntomouchtsis A, Patrikidou A, Christoforidou B, Andreadis C, Vahtsevanos K. Metastasis of a ductal breast carcinoma to the buccal mucosa of the mandible with tooth involvement. Oral Maxillofac Surg. 2012;16(4):377-81.
16- Kubota T. Metastatic models of human cancer xenografted in the nude mouse: the importance of orthotopic transplantation. J Cell Biochem. 1994;56(1):4-8.

17- Kumar GS, Manjunatha BS. Metastatic tumors to the jaws and oral cavity. J Oral Maxillofac Pathol. 2013;17(1):71-5.

18- Lee JH, Kim B, Jin WJ, Kim JW, Kim HH, Ha H, et al. Trolox inhibits osteolytic bone metastasis of breast cancer through both PGE2-dependent and independent mechanisms. Biochem Pharmacol. 2014;91(1):51-60.

19- Mano T, Wada N, Uchida K, Muraki Y, Nagatsuka H, Ueyama Y. Central adenoid cystic carcinoma of the mandible with multiple bone metastases: case report. J Oral Maxillofac Surg. 2010;68(2):446-51.

20- Nikfarjam L, Farzaneh P. Prevention and detection of Mycoplasma contamination in cell culture. Cell J. 2012;13(4):20312.

21- Ogütcen-Toller M, Metin M, Yildiz L. Metastatic breast carcinoma mimicking periodontal disease on radiographs. J Clin Periodontol. 2002;29(3):269-71.

22- Panossian M, Gady J, Natarajan E, Eisenberg E. Breast cancer metastatic to the mandible misdiagnosed as parotitis: report of a case. J Oral Maxillofac Surg. 2009;67(6):1333-7.

23- Pasupula AP, Dorankula SP, Thokala MR, Kumar MP. Metastatic follicular thyroid carcinoma to the mandible. Indian J Dent Res. $2012 ; 23(6): 843$.

24- Reyes Court D, Encina S, Levy I. Prostatic adenocarcinoma with mandibular metastatic lesion: case report. Med Oral Patol Oral Cir Bucal. 2007;12(6):E424-7.

25- Sasaki A, Nishiyama A, Alcalde RE, Lim DJ, Mese H, Nakayama $S$, et al. Effects of bisphosphonate on experimental jaw metastasis model in nude mice. Oral Oncol. 1999;35(5):523-9.

26- Sasaki A, Yoneda T, Terakado N, Alcalde RE, Suzuki A, Matsumura T. Experimental bone metastasis model of the oral and maxillofacial region. Anticancer Res. 1998;18(3A):1579-84. 27- Singh T, Amirtham U, Satheesh CT, Lakshmaiah KC, Suresh TM, Babu KG, et al. Floor-of-mouth metastasis in colorectal cancer. Ann Saudi Med. 2011;31(1):87-9.

28- Söder B, Yakob M, Meurman JH, Andersson LC, Klinge B, Söder PÖ. Periodontal disease may associate with breast cancer. Breast Cancer Res Treat. 2011;127(2):497-502.

29- Van der Horst G, van der Pluijm G. Preclinical models that illuminate the bone metastasis cascade. Recent Results Cancer Res. 2012;192:1-31.

30- Vazifeh Mostaan L, Irani S, Rajati M, Memar B. Mandibular metastasis from follicular thyroid carcinoma: a rare case after twelve years. Arch Iran Med. 2013;16(9):557-9.

31- Wang CL, Yacobi R, Pharoah M, Thorner P. Ewing's sarcoma: metastatic tumor to the jaw. Oral Surg Oral Med Oral Pathol. $1991 ; 71(5): 597-602$.

32- Will TA, Agarwal N, Petruzzelli GJ. Oral cavity metastasis of renal cell carcinoma: a case report. J Med Case Rep. 2008;2:313. 